\title{
Power Big Data Fusion Prediction
}

\author{
Liu Yan, Song Yu, Li Gang and Liang Weiqiang \\ School of Control and Computer Engineering, North China Electric Power University, Baoding 071003, China
}

\begin{abstract}
This paper is a research on the characteristics of power big data. According to the characteristics of "large volume", "species diversity", "sparse value density", "fast speed" of the power big data, a prediction model of multi-source information fusion for large data is established, the fusion prediction of various parameters of the same object is realized. A combined algorithm of Map Reduce and neural network is used in this paper. Using clustering and nonlinear mapping ability of neural network, it can effectively solve the problem of nonlinear objective function approximation, and neural network is applied to the prediction of fusion. In this paper, neural network model using multi layer feed forward network-BP neural network. Simultaneously, to achieve large-scale data sets in parallel computing, the parallelism and real-time property of the algorithm should be considered, further combined with Reduce Map model, to realize the parallel processing of the algorithm, making it more suitable for the study of the fusion of large data. And finally, through simulation, it verifies the feasibility of the proposed model and algorithm.
\end{abstract}

Key words: Power big data, fusion prediction, Map Reduce, BP neural network.

\section{Introduction}

Big data is another research hotspot after the cloud computing and the Internet of things. At present, there is no uniform definition for the concept of big data, but its "4V" [1] feature: "large volume", "species diversity", "sparse value density", "fast speed" is known. From this we can see that the volume of large data is very big, the amount of data is very much, and its data growth rate is very fast, showing a crazy explosive growth. And its value density is sparse, the type is diverse, there is the phenomenon of structured, semi-structured and unstructured data hybrid [2]. All these show that: at present, we are faced with the problem, how to dig out useful information from these large data [3].

Nowadays, we are in the era of power big data. In the power industry, a lot of data will be produced every day, these data show a significant feature of large data. In particular, the running state of the power equipment monitoring data [4], we need to find a way to dig out the useful information from these massive data [5]. In the power system, the power equipment [6]

Corresponding author: Liu Yan, graduate student, research field: logical combination of the visualization of large data. is the most basic component, the running state of the power equipment is directly related to the safety and reliability of the power system. Therefore, it is necessary to ensure the normal and safe operation of power equipment, and to do a good job in the safety maintenance of power equipment.

Prediction [7] is the core goal of the value mining of large data information. The operation condition monitoring of power equipment has the disadvantages of low utilization rate, no obvious effect and so on. The main reason is that the operation condition monitoring technology is not mature for the power system, understanding of the power equipment operating status, mostly relying on human experience, people pay less attention to the operation condition monitoring and maintenance of power equipment. In order to ensure the normal operation of electrical equipment, for effective management and maintenance of electrical equipment, to ensure the safety and reliability of the whole power system, it is necessary to monitor and forecast the power equipment [8]. From future trends by state power apparatus predictions, we can understand the future trend of the running status of equipment, analysis equipment in the operation of the potential failure of 
the potential threat [9].

It is well known that the prediction value and the actual results will be different, there is a certain deviation, so to find appropriate forecasting method is very important. A suitable forecasting model and the corresponding algorithm can avoid the influence factors unnecessary, and reduce the error value to the maximum degree. In the field of power system, this paper presents a new method for the fusion of electric power data.

\section{Application of BP Neural Network Algorithm}

Artificial neural network [10] can be referred to as "neural network", a kind of information processing mathematical model which is established by the way of information processing of human brain. It consists of mutual connection of a large number of neuron node of the network structure, each node represents a specific output function, which is called the excitation function or the conversion function, it can complete the information input, processing and output.

Artificial neural network can be divided into many kinds according to the difference of network structure and function. At present, the use of more is the BP neural network.

BP neural network refers to some use error back-propagation algorithm multilayer feedforward neural network. BP neural network uses the typical error back propagation algorithm, which has the ability of learning and memory, and it can be infinitely approximating any continuous nonlinear function. It has been proved that it has a good ability of nonlinear approximation. Its traditional algorithm consists of two processes [11]: signal forward propagation and error back propagation. The iterative correction formula [12] of the weight $\mathrm{W}$ of the algorithm is:

$$
\omega(k+1)=\omega(k)-\Delta \omega=\omega(k)-\left.\eta(\partial E / \partial \omega)\right|_{\omega=\omega(k)}
$$

where the value of $\omega$ is $0,1,2 \ldots, \Delta \omega$ is the weight of the $k$-th iteration, the threshold value correction amount:

$$
\Delta \omega=\left.\eta(\partial E / \partial w)\right|_{w=w(k)}
$$

Where $\partial E / \partial w$ represents the error function, $E$ is biased to the weight of $w . k$ is the Iteration number. $\boldsymbol{r}$ is the learning rate.

At present, many scholars have applied artificial neural network technology to forecast research field, and have achieved good results, but the relevant research in the field of electric power data is not deep enough. In this paper, the improved neural network model is applied to the field of large data fusion prediction. Using neural network clustering and nonlinear mapping ability to solve nonlinear objective function approximation problem, by fusing multiple attribute parameters of the same object, the correlation between them is obtained.

The BP neural network model is applied in the power of big data fusion prediction research, and selects three layer network structure, including input layer, hidden layer and output layer, as shown in Fig. 1. The number of neurons in the input layer is set to $\mathrm{N}$, which corresponds to the $\mathrm{N}$ input attribute of the object. Transfer function using linear identity:

$$
f(x)=x
$$

Hidden layer neurons are M. According to the actual situation of the specific input to determine the number of neurons, transfer function using Sigmoid excitation function:

$$
f(x)=\frac{1}{1+e^{-c u}}
$$

where $c$ represents the tilt parameter. The number of neurons in the output layer is set to one, and the output of the network corresponds to the power value in the power big data fusion prediction.

BP neural network learning training flow chart is shown in Fig. 2.

The BP neural network model is applied to the research of the large data fusion prediction:

Step 1: Input the various attributes of the object that need to be fused to forecast processing, these parameters are about to be input sample. For example, the prediction power of wind farm, the input should be attribute parameters of wind angle, impeller speed, 


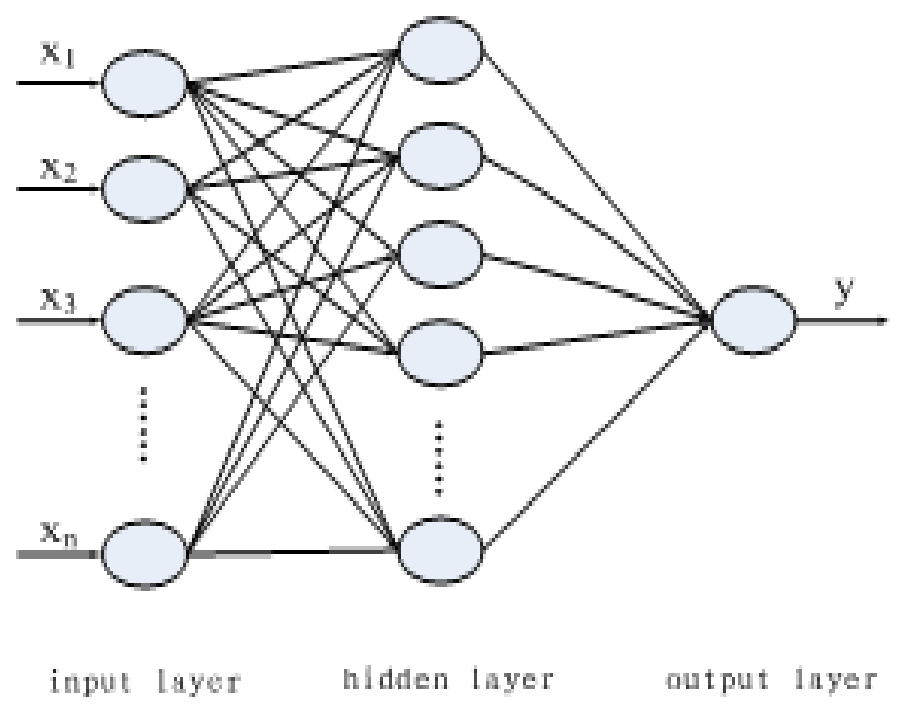

Fig. 1 Example of BP neural network model.

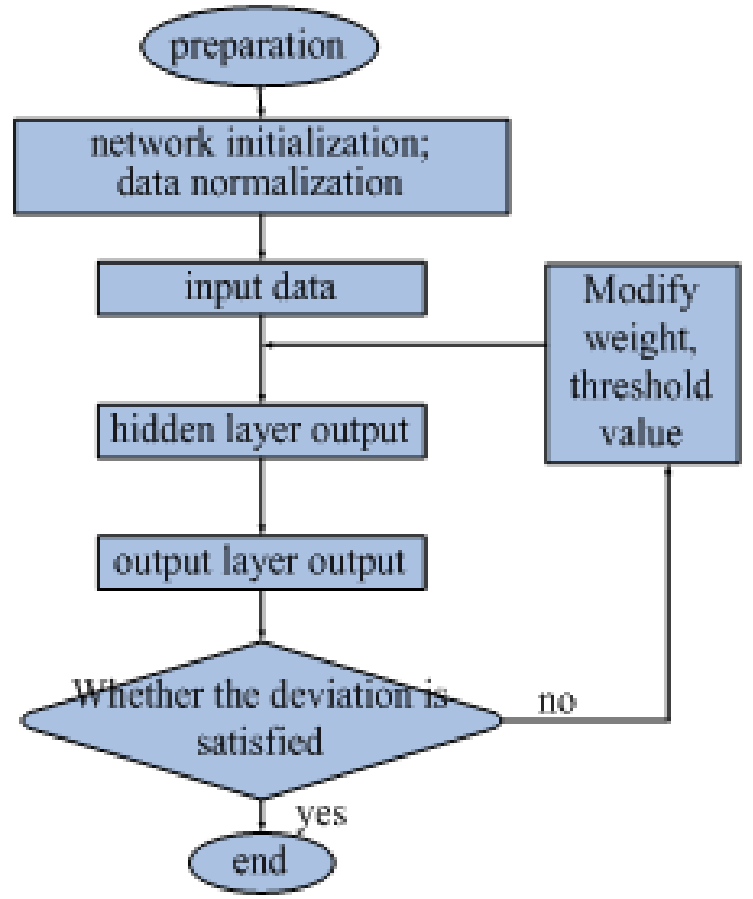

Fig. 2 BP neural network learning training flow chart.

engine temperature, phase voltage, generator speed, motor frequency etc.;

Step 2: Select the final attribute parameter to be used as the output sample. For example, the object is the wind farm, then the sample value of the power generation is selected as the output;

Step 3: Using neural network model to learn the sample;
Step 4: The simulation data of each attribute parameter is input to the trained neural network;

Step 5: Get the final attribute (the final prediction of wind farm is power generation) forecast value.

The flow chart of BP neural network model applied to the prediction of power big data fusion is shown in Fig. 3.

\section{Parallel Algorithm Based on Map Reduce}

Power big data on the requirements of the algorithm is very high, it requires that the algorithm has the characteristics of "parallel" and "real time". Reduce Map model has the ability to parallel processing algorithm, and it can be used for large-scale data sets of parallel computing, to meet the requirements of the parallel processing power of large data.

Combining Map Reduce and BP neural network, the algorithm can be used in parallel processing, which can be more suitable for the fusion of large data. Map Reduce can be divided into two parts: mapping and reduction. Map mechanisms are responsible for dividing large data sets for each node in the network, each node is responsible for the operation of their own 


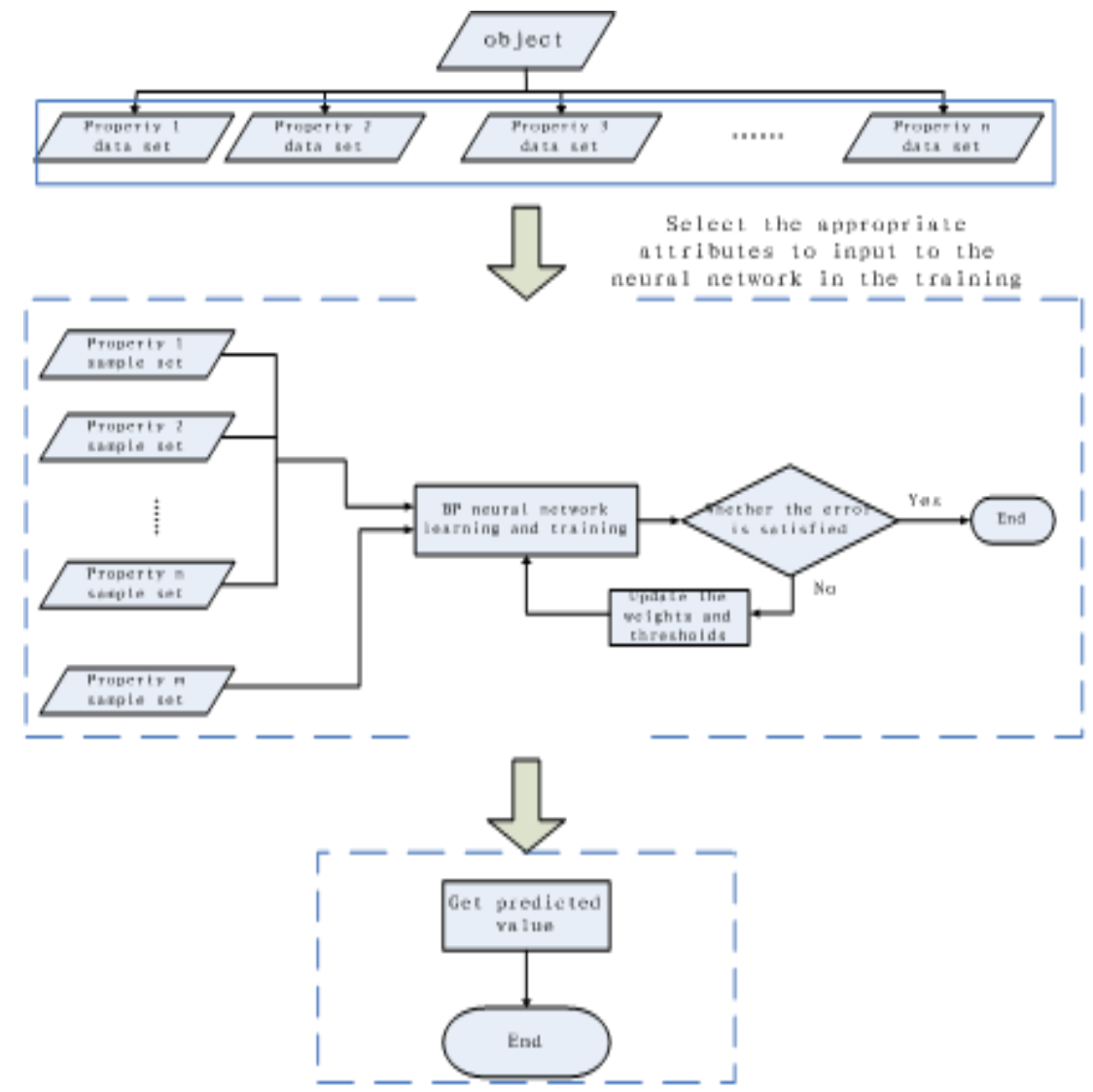

Fig. 3 Flow chart of BP neural network fusion prediction.

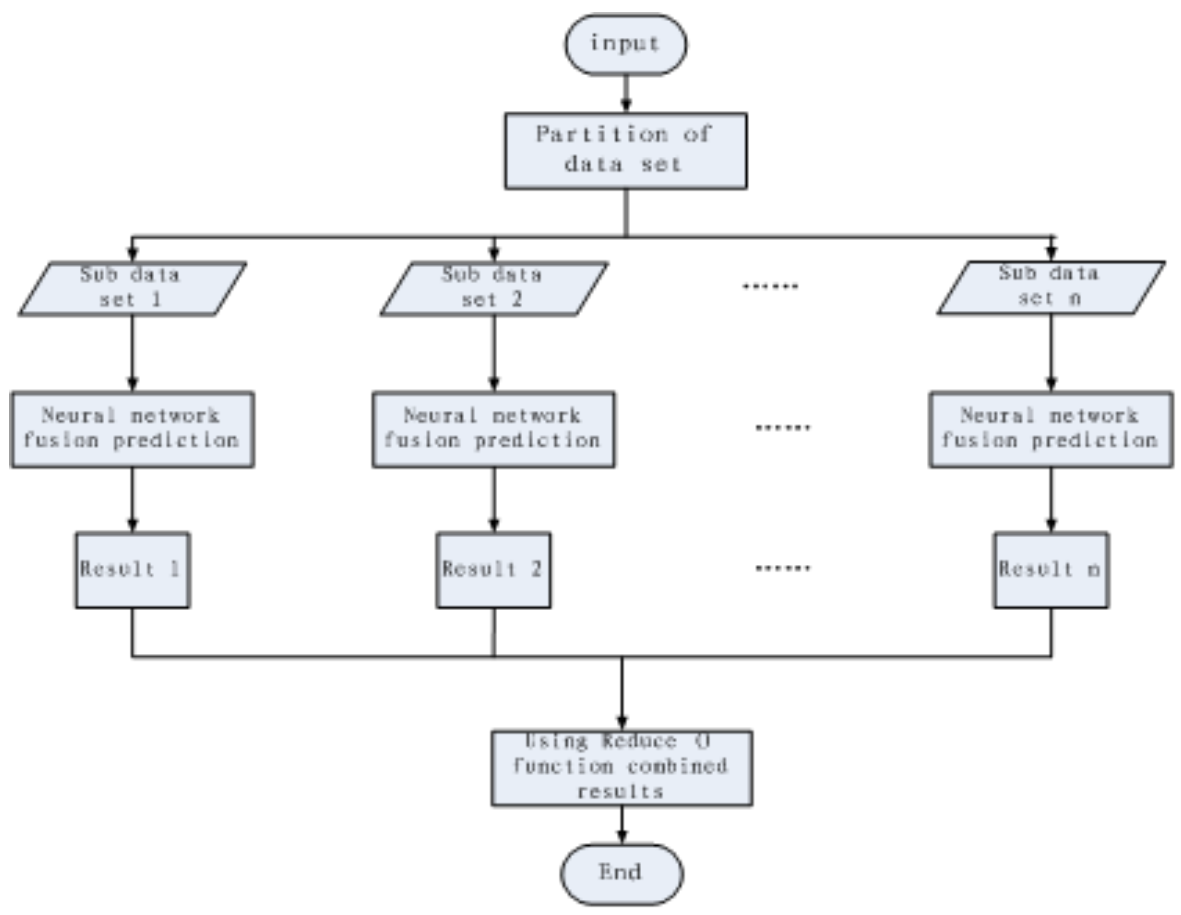

Fig. 4 Schematic diagram of parallel algorithm based on Map Reduce. 
part, which is the use of $\mathrm{BP}$ neural network to complete the data fusion prediction. Then the Reduce mechanism is responsible for merging the results of each node, so as to get the final result. The flow chart of parallel processing using Map Reduce is shown in Fig. 4.

\section{Results and Discussion}

The simulation software is Matlab. A wind farm is used as the experimental object. State parameters include wind direction, generator temperature, active power, wind speed and so on.

A part of these state parameters are now selected, the normalized data as input data and power normalized data as output samples, then train the neural network. Finally, after the input of the normalized simulation data, the predicted value of the power generation power is obtained, and compared with the true value, the obtained figure is shown in Fig. 5.

The red line is the predicted value of the BP neural network training, the blue line is a real value, the number of simulation samples represented by the horizontal coordinates, vertical coordinates represents their numerical values. As can be seen from the figure, the BP neural network can be used to approach the true value very well, and it can achieve a good result of data fitting.

Therefore, we can use this method to predict the future power of the wind turbine. Fig. 6 shows the power forecast for a certain period of time in the future. Fig. 7 shows the trend of future power forecast.

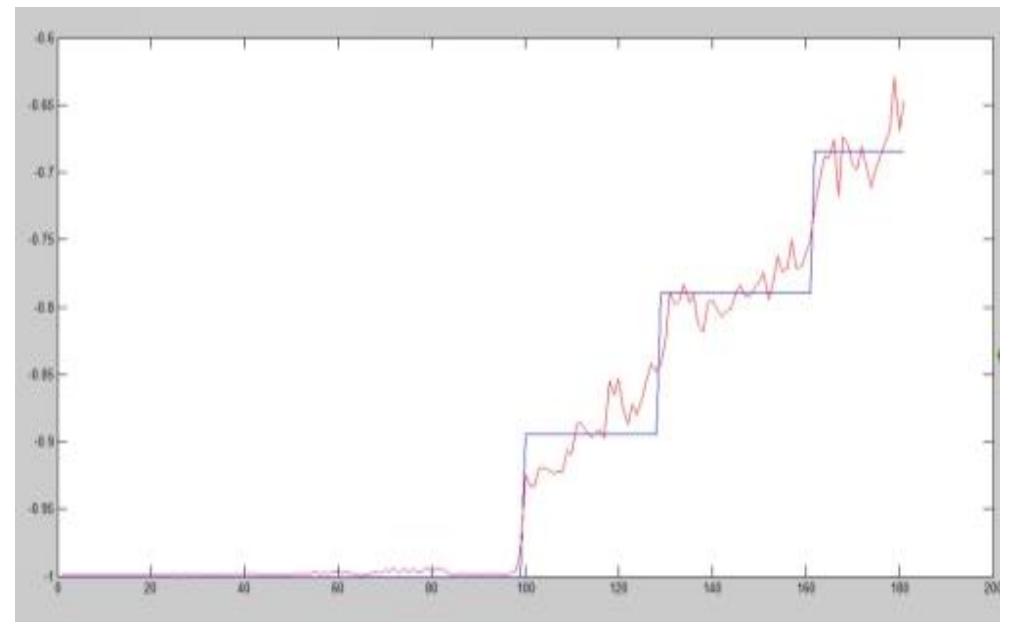

Fig. 5 Simulation results.

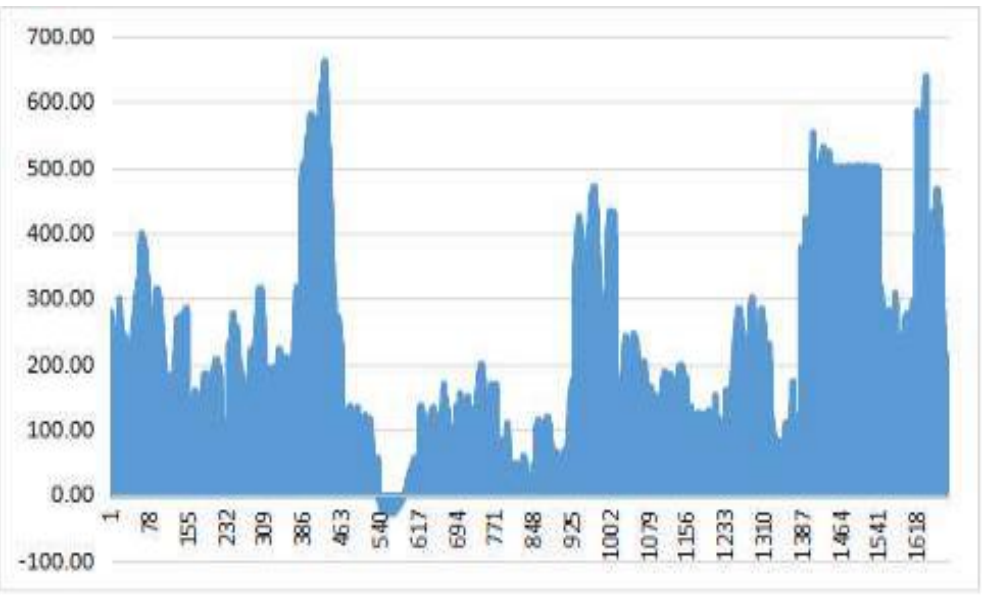

Fig. 6 Future power forecast. 


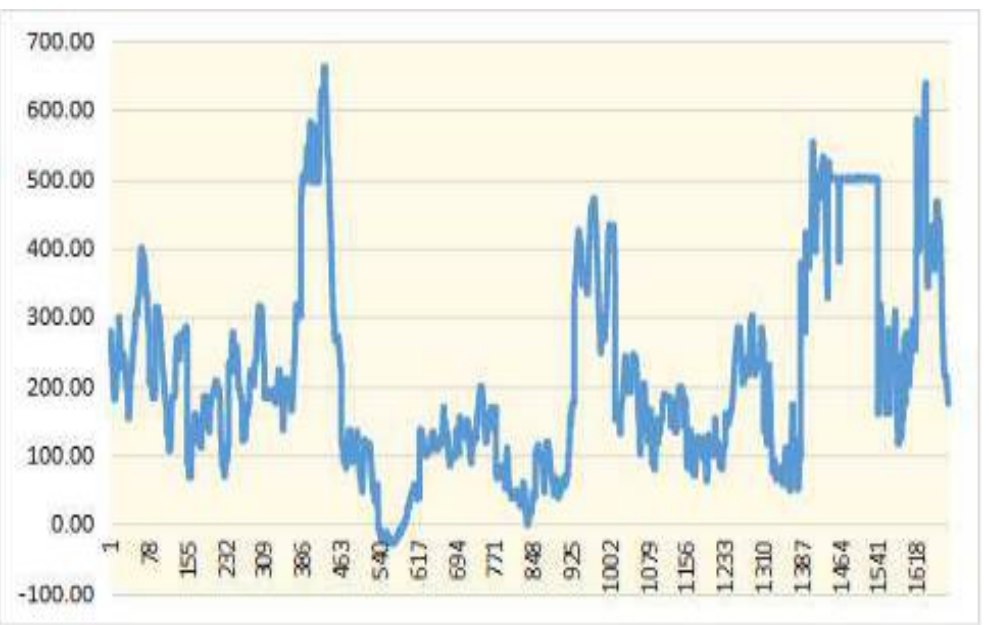

Fig. 7 Trend of power prediction.

\section{Conclusions}

According to the characteristics of large power data, this paper proposes a fusion prediction model which is suitable for large power data. Using clustering and nonlinear mapping ability of neural network, and its good function approximation ability, the output value of the target output can be obtained, so as to achieve the ultimate goal of fusion prediction. At the same time, combined with the Reduce Map model, the parallel processing algorithm is realized. Method of combining BP neural network and Map Reduce, to meet the processing requirements of the "real-time" and "Parallelism" of power big data. So the prediction algorithm fusion can suitable for large power application of data field. Finally, a numerical example is used to verify the feasibility of the proposed method.

\section{Acknowledgements}

This work is supported by National Natural Science Foundation of China (51407076) and the Natural Science Foundation of Hebei Province (F2014502050), The Higher Education Scientific Research Project of Hebei Province (Z2013007), the Fundamental Research Funds for the Central Universities (2015ZD28).

\section{References}

[1] Wang, D. W. 2012. "Basic Framework and Key
Technology for a New Generation of Data Center in Electric Power Corporation based on Cloud Computation." Automation of Electric Power System 36 (11): 67-71.

[2] Song, Y. Q., Zhou, G. L., and Zhu, Y. L. 2013. "Present Status and Challenges of Big Data Processing in Smart Grid.” Power System Technology 37 (4): 927-35.

[3] Meng, X. F., and Ci, X. 2013. "Big Data Management: Concepts, Techniques, Challenges." Journal of Computer Research and Development 50 (1): 146-69.

[4] Peng, X. S., Deng, D. Y., Cheng, S. J., Wen, J. Y., Li, Z. H., and Niu, L. 2015. "A Key Technology for Smart Grid Applications of Power Data." The Chinese Journal of Electrical Engineering 3: 503-11.

[5] Yue, Y., Zhang, X. J., and Gao, Y. D. 2015. "Research of Power Large Data Technology System Based on Hadoop." Electric Power and Energy 1: 16-20.

[6] Yan, L. C., Li, Y. X., Li, B. C., and Zhao, Z. Y. 2013. "The Opportunity and Challenge of the Power Big Data Facing." Electric Power Information 4: 1-4.

[7] Zhao, Y. S., and Liu, H. H. 2014. "Research on the Application of Big Data Technology in the Electric Power Industry.” Telecommunications Science 1: 57-62.

[8] Xiong, Z. B. 2011. "ARIMA Fusion Neural Network Based on the RMB Exchange Rate Prediction Model." Quantitative Economic and Technical Research 6: 64-76.

[9] Fang, Y. 2011. "Prediction in BP Neural Network Fusion Technology." Coal Technology 8: 107-9.

[10] Zeng, Q. S., Quan, S. P., and Jin, Z. Q. 2011. "Short Term Traffic Flow Forecasting on Fusion of ARIMA and BP Neural Network." Journal of Zhengzhou University (Engineering Science) 4: 60-3.

[11] Wachter, B., Kroening, D., and Ouaknine, J. 2013. "Verifying Multi-threaded Software with Impact." Formal Methods in Computer-Aided Design (FMCAD): 210-7. 
[12] Chen, Y., Meng, Z., Ma, S. Q., and Bao, C. C. 2015. "High-Resolution Azimuth Estimation Algorithm Based on Data Fusion Method for the Vector Hydrophone Vertical Array." Chinese Journal of Acoustics 3: 312-24. 